\title{
Chocolate, biscuits and fruit bars: effects on appetite and mood
}

\author{
K. M. Appleton, P. P. McKeown and J. V. Woodside \\ Queen's University, Belfast, Belfast, BT9 5BP, UK
}

Various historical work and anecdotal comments suggest that a small portion of good quality chocolate at the end of a meal may aid digestion, reduce subsequent appetite and aid appetite control ${ }^{(1)}$. Recent research also suggests various health benefits from the consumption of chocolate high in cocoa concentrations ${ }^{(2,3)}$. This study aimed at investigating the impact of a small portion of good quality chocolate on short-term appetite. Effects of small portions of good quality chocolate were compared to effects of similar small portions of poor quality chocolate, biscuits, fruit bars and nothing.

The study was conducted using a repeated measures design and preloading procedure, where small portions of the four different foods were provided to twenty participants at four time points across the day. Lunch intake, evening meal intake and perceptions of hunger, mood, liking and acceptability of all foods were subsequently measured. All participants undertook all five conditions on separate days, one week apart.

At lunch intake, participants consumed significantly less following good quality chocolate, poor quality chocolate and biscuits than following nothing $(F(4,76)=2.52, P=0.05)$, but no differences were found between conditions at tea intake, in cumulative (lunch + tea) intake or total (lunch + tea + food portions) intake (largest $F(4,76)=1.99, P=0.10$ ). Across the morning, participants also reported significantly lower hunger, desire to eat and prospective consumption and significantly greater fullness following good quality chocolate, biscuits and fruit bars compared to no food (smallest $t(19)=2.19, P=0.04$ ), but no effects were found across the rest of the day (largest $F(4,76)=1.64, P=0.17)$. Across the day, participants reported significantly lower alertness following no food compared to following all foods, and significantly higher tiredness following no food and poor quality chocolate compared to all other foods (smallest $F(4,76)=3.74, P=0.01)$. No differences were found between all foods in measures of pleasantness, liking and satisfaction (largest $F(3,54)=2.36, P=0.08)$, but all other foods were rated more familiar than fruit bars $F(3,54)=17.93, P<0.01)$. In measures of acceptability, participants reported no differences between all foods in how content they would be to consume them $($ largest $F(3,57)=2.29$, $P=0.09)$, but participants reported being significantly more likely to consume and buy poor quality chocolate and biscuits than good quality chocolate and fruit bars (smallest $F(3,57)=3.75, P=0.02$ ).

These findings suggest that a small portion of good quality chocolate at the end of a meal has limited effects on appetite and may be unlikely to aid appetite control. This absence of effects suggests that effects that have previously been reported are unlikely to be a result of the cocoa content or any other specific component of good quality chocolate ${ }^{(1)}$. The lack of differences in total energy intake, however, also suggest that none of the food portions were found to impact negatively on appetite or subsequent intake, and the effects on mood suggest that all foods may aid alertness and reduce feelings of tiredness. The lack of differences in liking and acceptability may also aid the promotion of consumption of good quality chocolate and fruit bars for health reasons ${ }^{(2,3)}$.

1. Dillinger TL, Barriga P, Escarcega S et al. (2000) Food of the gods: cure for humanity? A cultural history of the medicinal and ritual use of chocolate. J Nutr 130, 2057S-2072S.

2. Faridi Z, Njike VY, Dutta S et al. (2008) Acute dark chocolate and coca ingestion and endothelial function: a randomized controlled crossover trial AJCN 88, 58-63

3. Grassi D, Lippi C, Necozione S et al. (2005) Short term administration of dark chocolate is followed by a significant increase in insulin sensitivity and a decrease in blood pressure in healthy persons. AJCN 81, 611-614. 